\title{
Nanomess- und Nanopositioniergeräte für die nanometergenaue Positionierung, Messung und Bearbeitung von Oberflächen und Strukturen
}

\author{
Schwerpunkt: B5 Nanomessmaschinen
}

Prof.-Dr.-Ing. habil. Eberhard Manske, Prof.-Dr.-Ing. habil. Gerd Jäger, PD Dr.-Ing. habil. Roland FüßI, DI Felix Balzer, DI Torsten Machleidt

Anschrift: TU Ilmenau, Institut Prozessmess- und Sensortechnik, PF 100565, 98684 Ilmenau, Tel.-Nr. 03677 692822, E-Mail-Adresse: eberhard.manske@tu-ilmenau.de

\section{Kurzfassung}

Nanomess- und Nanopositioniergeräte sind für die nanometergenaue Positionierung und Messung z.B. von Oberflächen und Strukturen, von mechanischen und optischen Präzisionsteilen sowie für die Materialanalyse erforderlich. Die Notwendigkeit, immer kleinere Objekte in immer größeren Raumbereichen zu beherrschen, ergibt sich durch den anhaltenden Fortschritt der Mikro- und Nanotechnologien, insbesondere der Halbleiterindustrie, der Ultrapräzisionsbearbeitung und vieler damit verbundener Technologien. Um die immer größere Breite und Komplexität an Messaufgaben in der Mikro- und Nanotechnologie erfüllen zu können, ist ein Multisensoransatz auch für Nanopositionier- und Nanomessmaschinen sinnvoll. Neben einer ganzen Reihe von optischen, taktilen und Rasterkraftsensoren werden erste Untersuchungen zu Nanotools für die Nanobearbeitung von Oberflächen mit den Möglichkeiten der Nanopositionier- und Nanomessmaschinen vorgestellt.

\begin{abstract}
Nanomeasuring and nanopositioning devices are necessary for positioning and measuring surfaces and structures, mechanical and optical precision parts with nanometre precision.

The ability of measuring ever smaller objects in ever larger spatial areas is motivated by the permanent progress of micro- and nanotechnologies, especially of semiconductor industries, ultra-precision technologies and many other associated technologies. To meet the increasing demands concerning the ever greater complexity and variety of the measurement tasks in micro- and nanotechnologies it is reasonable to implement a multisensory approach also in nanopositioning and nanomeasuring machines. Besides a set of optical, tactile and scanning force probes some first investigations regarding the nanofabrication of surfaces by means of nanopositioning and nanomeasuring machines are presented.
\end{abstract}

\section{Einleitung}

Im Bereich der Nanotechnologien werden immer neue Verfahren zur Strukturierung von Oberflächen entwickelt. Die rasante Entwicklung der Halbleiterindustrie, entsprechend der International Technological Roadmap of Semiconductors [ITRS], erreicht gegenwärtig DRAM-1/2Pitch-Breiten von $<40 \mathrm{~nm}$.

Durch Funktionalisierung von Oberflächen mit Nanostrukturen können die intrinsischen Eigenschaften von Werkstoffen verändert werden bzw. aufgrund ihrer Dimension einen besonderen Effekt hervorrufen. Geräte und Ausrüstungen für solche Technologien verfügen meist nur über Bearbeitungsbereiche von einigen 100 Mikrometern. Der Bedarf an einer skalenübergreifenden Nanopositionier- und Nanomesstechnik mit Messbereichen von mehreren Zentimetern und gleichzeitiger Nanometerpräzision wird immer größer. Die Nanopositionier- und Nanomessmaschine, entwickelt durch die TU IImenau und SIOS Meßtechnik GmbH, stellt hier ein leistungsfähiges Werkzeug dar.

Die Vielzahl der Messaufgaben im Submikro- und Nanometerbereich kann allerdings nicht mit Hilfe eines einzelnen Antastsensors, bzw. Antastprinzips verwirklicht werden. Gegenwärtig stehen optische, taktile und Rasterkraft-Punktsensoren sowie optische Flächensensoren zur 2,5D-Messung zur Verfügung. Zur wirklichen 3D-Messung gibt es eine ganze Reihe taktiler 3D-Mikrotaster. Um die unterschiedlichen Sensorprinzipien bezüglich ihrer unterschiedlichen Vor- und Nachteile optimal einzusetzen wurde eine Multisensoranordnung auf der Basis eines Mikroskoprevolvers in die Nanopositionier- und Nanomessmaschine integriert.

Neben dem Einsatz unterschiedlicher Nanosensoren ist auch die Integration von Nanotools in die Nanopositionier- und Nanomessmaschine möglich. So können Rastersondentechniken, wie das Nanoscratching oder die Nanooxydation verwendet werden, um großflächige Nanolithographie im Messvolumen der Nanopositionier- und Nanomessmaschine von $25 \mathrm{~mm}$ x $25 \mathrm{~mm}$ x $5 \mathrm{~mm}$ zu realisieren. 


\section{Nanopositionier- und Nanomessmaschinen}

Höchste Genauigkeit im Nanometerbereich kann nur durch die konsequente Anwendung fehlerminimaler Messanordnungen erreicht werden. Für eine dreidimensionale Messung ist es deshalb erforderlich, das Abbe-Komparatorprinzip, die fluchtende Anordnung von Prüfling und Normal, ebenfalls dreidimensional anzuwenden. Auf diesem Prinzip basieren inzwischen eine ganze Reihe von Maschinen, wie z.B. die „Molecular Measuring Machine“ des NIST der USA [4], die "Sub-atomic Measuring Machine“ [5], die Nanopositionier- und Nanomessmaschine (NPM-Maschine) der TU IImenau [7] und die Ultraprecision CMM „Isara 400“ [8].

Hierbei müssen sich die drei Messachsen der drei orthogonalen Koordinatenachsen zu jeder Zeit im Antastpunkt des Antastsystems schneiden. Das wird dahingehend realisiert, dass drei PlanspiegelLaserinterferometer orthogonal zueinander fest angeordnet sind und der Messtisch aus einer Spiegelecke zur Reflektion der Laserstrahlen besteht (vgl. Bild 1).

Zusätzlich wird in der NPM-Maschine die Verkippung der Spiegelecke kontinuierlich gemessen und mit einer Reproduzierbarkeit von < 0,2" aktiv ausgeregelt. Damit erreicht man eine weitere Verkleinerung des Abbe-Fehlers, indem sowohl der Abbeoffset als auch die Winkelverkippung in allen drei Achsen minimiert wird:

$\Delta \mathrm{l}_{\mathrm{i}}=\mathrm{l}_{\text {offset }} \cdot \sin \alpha_{\mathrm{i}} \rightarrow 0$ für $\mathrm{i} \in\{\mathrm{x}, \mathrm{y}, \mathrm{z}\}$.

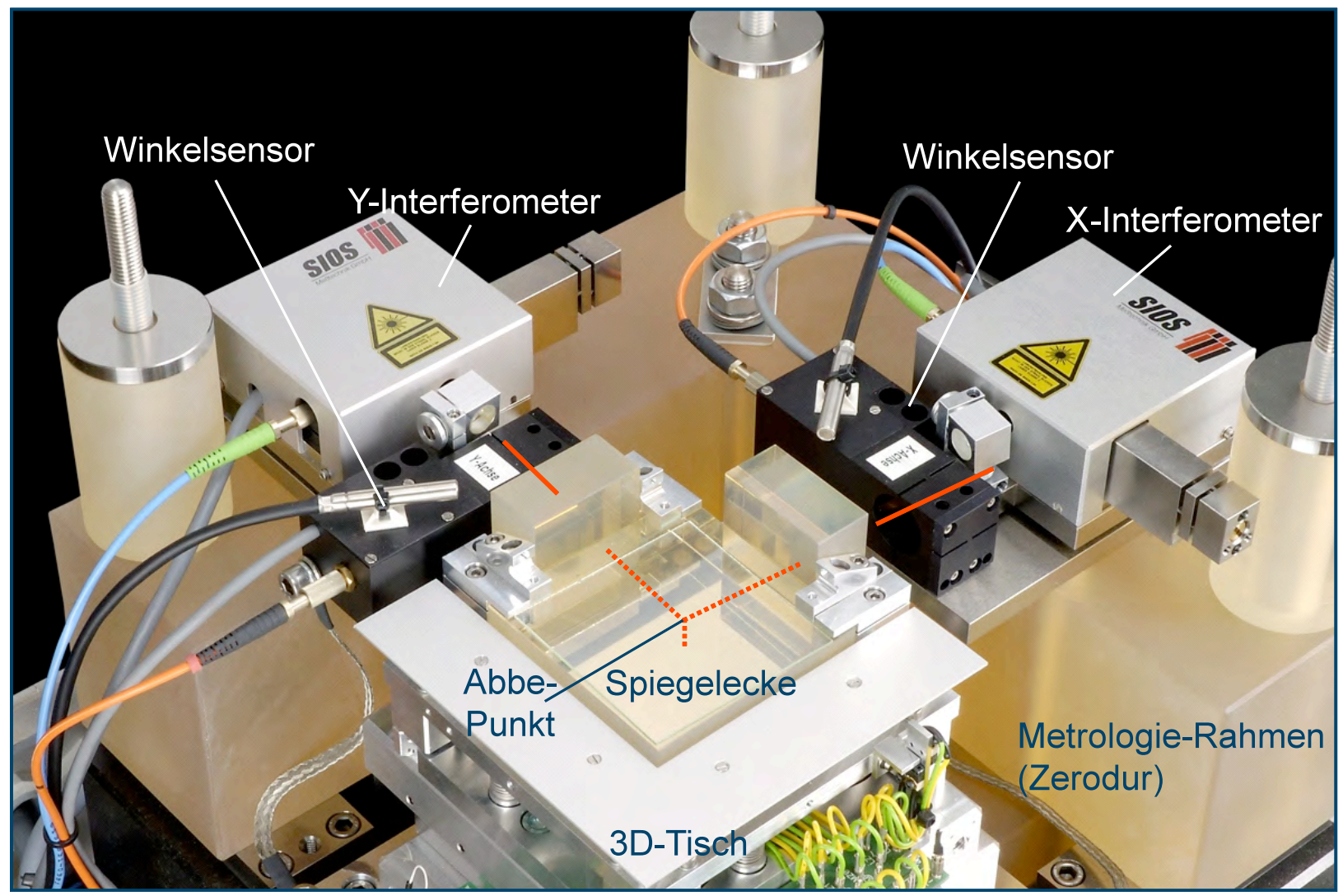

Abbildung 1: Grundaufbau der Nanopositionier- und Nanomessmaschine

Die Antastung der Messoberfläche erfolgt mit raumfest angeordneten „Nullpunkt“-Sensoren. Diese detektieren das Messobjekt nur innerhalb eines äußerst kleinen Messbereiches (<1 1 m) mit Auflösungen von kleiner als einem Nanometer. Durch die konstruktive Gestaltung der Messmaschine ist es möglich, unterschiedlichste Antastsensoren zu nutzen und so den Anforderungen des Messobjektes, insbesondere hinsichtlich lateraler Auflösung, Messgeschwindigkeit, mechanischer oder berührungsloser Antastung gerecht zu werden. Neben konventionellen Sensoren (z.B. Rastersondenmikroskopen) 
wurden eine ganze Reihe eigener Antastsensoren mit unterschiedlichsten messtechnischen Eigenschaften entwickelt.

\section{Antastsensoren}

Optische Laserfokussensoren ermöglichen durch die optisch-berührungslose Messung schnelle und hochgenaue Linien- und Oberflächenscans. Die Hologram-Laser-Unit detektiert dabei ein Fokusfehlersignal, wenn sich das zu messende Objekt nicht im Fokuspunkt des Messobjektives befindet (Bild 2) [8]. Da der Sensor nur punktförmige Messungen ausführen kann, wurde eine zusätzliche CCDKamera zum schnellen Auffinden der zu messenden Strukturen integriert.

Durch eine empfindliche Signaldetektion kann eine Auflösung in vertikaler Richtung von $<1 \mathrm{~nm}$ erreicht werden. Bei der verwendeten Wellenlänge von $650 \mathrm{~nm}$ und einer Apertur von 0,55 beträgt die laterale Auflösung etwa 0,6 $\mu \mathrm{m}$. Insbesondere bei relativ monotonen Oberflächen können Scangeschwindigkeiten bis zu $5 \mathrm{~mm} / \mathrm{s}$ bei Messunsicherheiten von 1-2 $\mathrm{nm}$ erreicht werden. Der große z-Bereich der NPMMaschine von $5 \mathrm{~mm}$ kann mit Hilfe eines Objektives mit einem Arbeitsabstand von $10 \mathrm{~mm}$ problemlos ausgeschöpft werden. Damit sind Stufenhöhenmessungen bis $5 \mathrm{~mm}$ ohne Kollisionsgefahr mit Nanometerpräzision möglich.

Optische Sensoren ermöglichen, da sie berührungslos arbeiten, hohe Scangeschwindigkeiten, weisen allerdings auch einige spezifische Nachteile auf. Es können Beugungserscheinungen, sogenannte „Überschwinger“, an Kanten auftreten, die zu großen Fehlinterpretationen der wirklichen Geometrie führen können. Bei der Messung auf unterschiedlichen Materialien kann es durch optische Phasensprünge zur Verfälschung bei Stufenhöhenmessungen kommen. Die laterale Auflösung ist ebenfalls beugungsbedingt auf $0,5-1 \mu \mathrm{m}$ begrenzt.

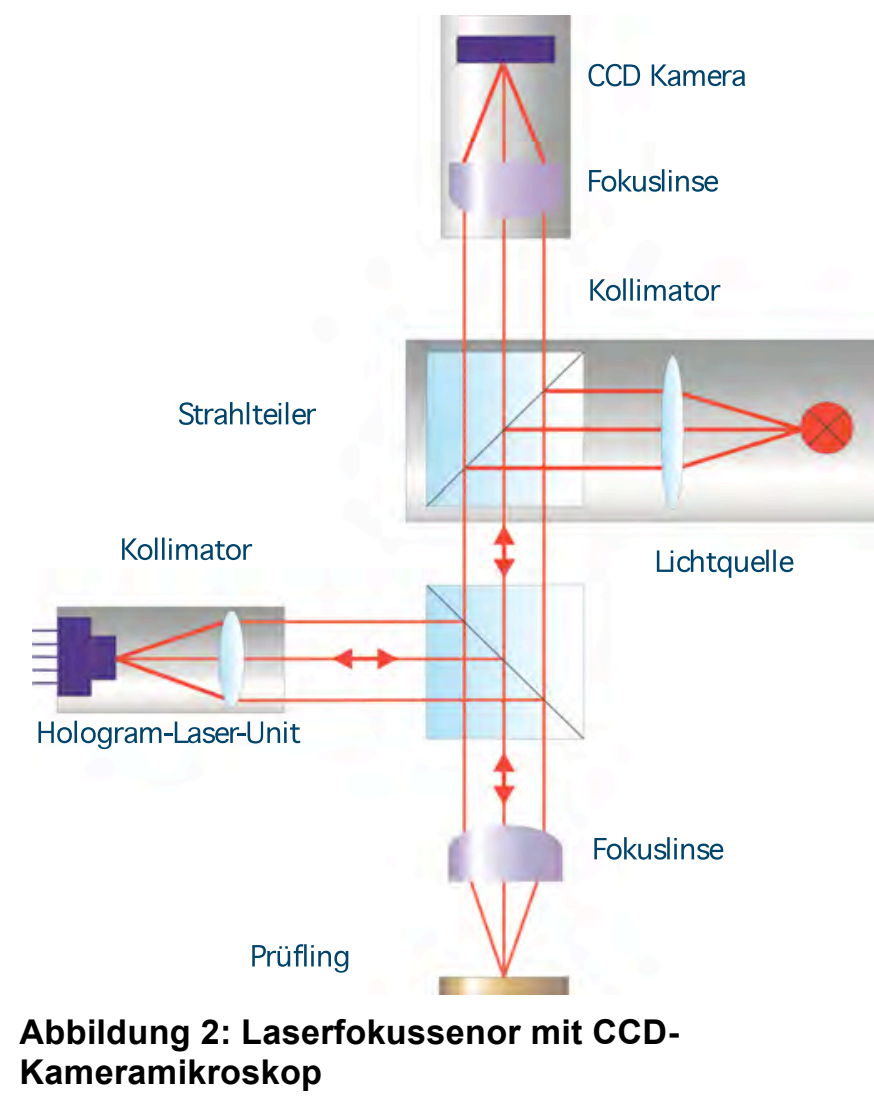

Taktile Messverfahren gelten als rückführbare Messverfahren. Sie weisen weder Beugungserscheinungen noch Phasensprünge auf. Allerdings können die verwendeten Messkräfte bereits Bearbeitungsspuren auf der zu messenden Oberfläche hinterlassen. Taktile Tastschnittsensoren besitzen typischerweise Tastnadeldurchmesser von $4 \mu \mathrm{m}$, die damit die Grenze der lateralen Auflösung bestimmen. Die Verfahrgeschwindigkeit ist infolge des dynamischen Verhaltens der Tastnadel auf wenige $100 \mu \mathrm{m} / \mathrm{s}$ beschränkt.

Für höhere laterale Auflösungen können Rastersondentechniken mit Cantileverspitzen von <10 nm eingesetzt werden. Die Scangeschwindigkeiten sind hier natürlich ebenfalls begrenzt. Durch verschiedene Ansätze versucht man „High Speed“Rastersondenscans zu realisieren. Im KontaktModus wurden mit speziellen Regelalgorithmen Scangeschwindigkeiten bis $200 \mu \mathrm{m} / \mathrm{s}$ erreicht [9].

Um die unterschiedlichen Antastprinzipien zu vereinigen wurden Zusatzmodule für den Laserfokussensor entwickelt, die es ermöglichen, den Laserfokuspunkt auf die Rückseite einer Tastnadel eines Oberflächentastschnittsensors bzw. von Rastersondencantilevern zu fokussieren. Damit ist es möglich, die oben dargestellten Antastprinzipien auf der Basis des Laserfokussensors praktisch umzusetzen [8].

Eine Erhöhung der Messdatenerfassungsgeschwindigkeit ist mit Array-Sensoren möglich. Mittels Weißlichtinterferenzmikroskopie kann eine parallele Datenaufnahme von mehreren Millionen Messpunkten (je nach verwendeter CCD-Kamera) durch einen Höhenscan innerhalb von wenigen 
Sekunden realisiert werden [8]. Entsprechend der Objektivvergrößerung können dabei Messfelder, z.B. von $800 \mu \mathrm{m} \times 600 \mu \mathrm{m}$ (20-fach) bis $320 \mu \mathrm{m} \times 240 \mu \mathrm{m}$ (50-fach), mit einem Scan erfasst werden. Die Ermittlung der Höhendaten erfolgt mit der laserinterferometrischen Präzision der NPM-Maschine bis zu Stufenhöhen von etwa $3 \mathrm{~mm}$ (entsprechend des Arbeitsabstandes des Mirau-Interferometers).

Die Konsistenz der verschiedenen Antastsysteme für Messungen mit der NPM-Maschine wurde für Stufenhöhenmessungen von $7 \mathrm{~nm}$ bis $800 \mathrm{~nm}$ mit Hilfe von zertifizierten Stufenhöhennormalen nachgewiesen [10].

\section{Multisensoranordnung mit der NPM-Maschine}

Um einen kompakten Multisensoransatz zu erzielen, können die unterschiedlichen Sensoren auf einer gemeinsamen Plattform zusammengebracht werden (vgl. Abbildung 3). Eine Zusammenführung der einzelnen Sensorkonfigurationen kann auf der Basis eines Mikroskoprevolvers erfolgen. Nach ersten Tests in einem externen Messaufbau wurde der Messrevolver in die NPM-Maschine integriert (vgl. Abbildung 5).

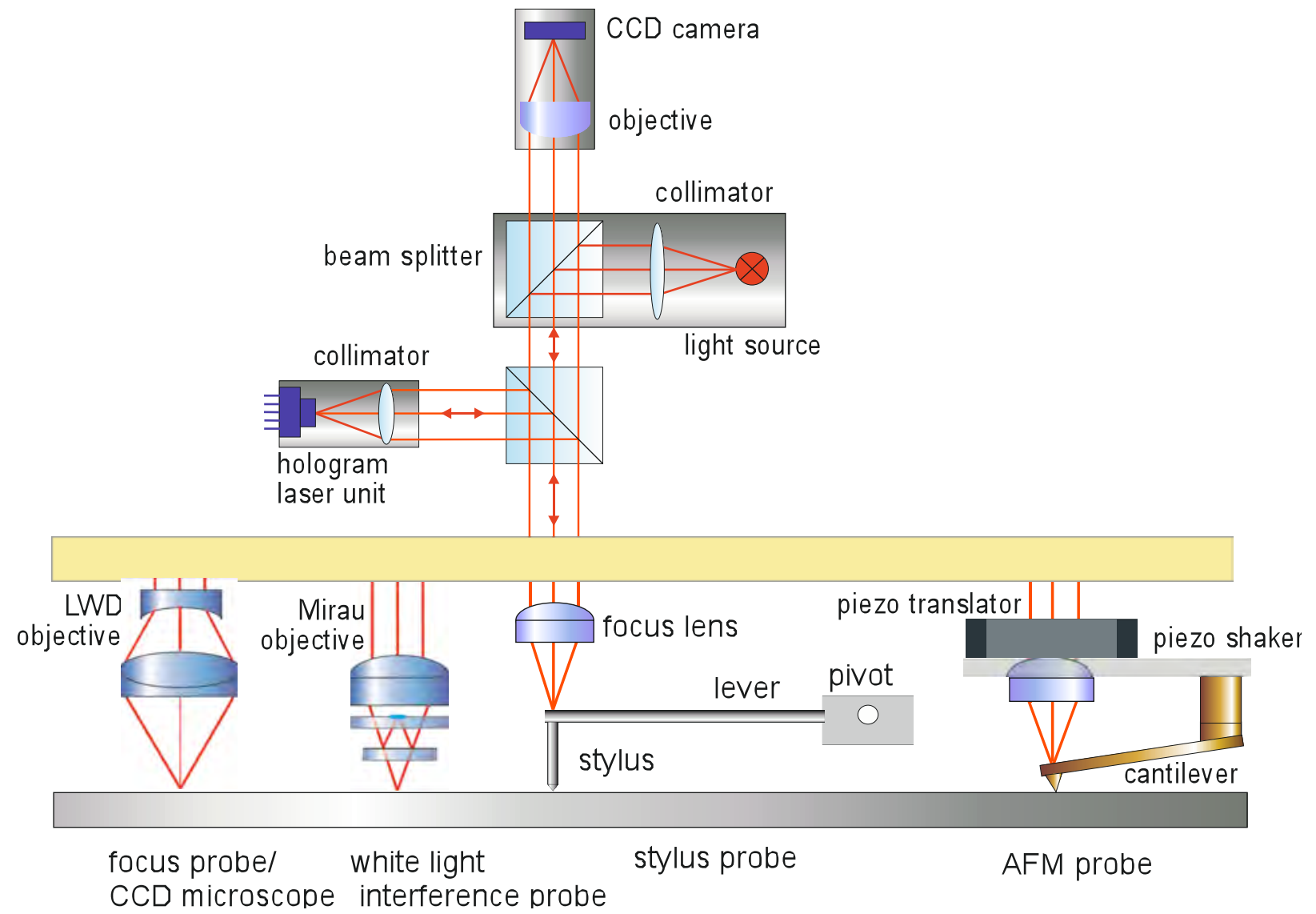

Abbildung 3: Multisensorplattform für die NPM-Maschine

Mit diesem Ansatz ist man nun in der Lage, einen Prüfling mit verschiedenen Messsystemen zu messen. Beispielsweise können unterschiedliche Areale eines Messobjektes unterschiedliche Anforderungen an die laterale Messauflösung, die Kantendetektion oder das Erfordernis der berührungslosen Messung u.ä. aufweisen. Die Sensordaten unterschiedlicher Sensoren können nur dann nanometergenau fusioniert werden, wenn der Antastort aller Sensoren übereinstimmt bzw. nanometergenau bekannt ist. Die Reproduzierbarkeit des Mikroskoprevolvers ist für diesen Anspruch nicht ausreichend. Aus diesem Grund wird bei der Anwendung des Revolversystems mit Hilfe von Bezugsmarken gearbeitet. Diese Bezugsmarken müssen für die unterschiedlichen Sensoren gleichermaßen detektierbar sein. Sie können auf dem Messtisch oder direkt auf dem Prüfling angebracht sein. Nach dem Umschalten des Revolvers zwischen unterschiedlichen Sensor muss die Lage dieser Bezugsmarken innerhalb des 
Maschinenkoordinatensystems wieder neu ermittelt werden. Damit können die Koordinaten unterschiedlicher Messungen miteinander fusioniert werden.

Die Genauigkeit der Messdatenfusion ist nun (nur noch) von der Lagestabilität des Antastpunktes in einer Stellung des Revolvers während der Messung mit einem Sensor abhängig. Zum zweiten ist eine Langzeitstabilität der Lage der Bezugsmarken erforderlich. Zum dritten geht die Messunsicherheit bei der Bestimmung der Bezugsmarken mit unterschiedlichen Antastsensoren direkt in die Messunsicherheit der Datenfusion ein. Für letzteres ist es erforderlich, dass unterschiedliche Sensoren, z.B. der optische Laserfokussensor mit einer lateralen Auflösung von etwa 0,6 $\mu \mathrm{m}$ und der Rastersondensensor mit einem typischen Spitzenradius von $10 \mathrm{~nm}$ bei der Messung an einer Prüfstruktur trotz unterschiedlicher lateraler Auflösung, trotz unterschiedlicher physikalischer Prinzipien der Antastung und somit total unterschiedlichen Wechselwirkungen zwischen Sensor und Messstruktur den gleichen geometrischen Schwerpunkt ermitteln. Beispielsweise wurde an der BAM eine Kalibrierpyramide mit Nanomarkern entwickelt. Diese Nanomarker sind so gewählt, dass sie sowohl mit AFM-Methoden als auch mit konfokaler Mikroskopie gemessen werden können [11].

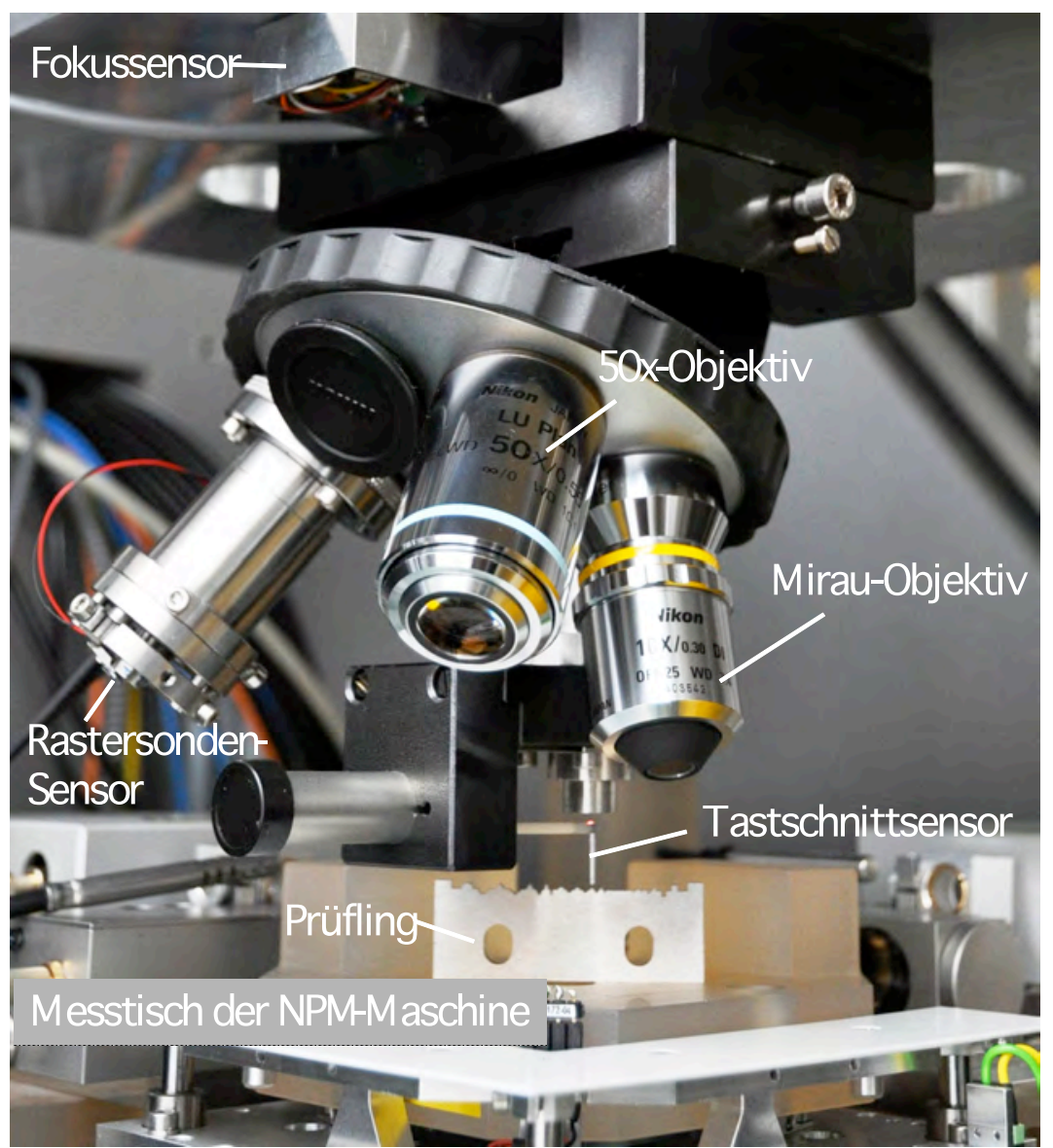

Abbildung 5: Messrevolver in der NPM-Maschine

Um diese Bezugsmarken-Technologie zuverlässig anwenden zu können, sind weitere intensive Untersuchungen zur Vergleichbarkeit und Langzeitstabilität erforderlich.

\section{Neue Messstrategien für nanotechnologische Anwendungen}

Aus den immensen messtechnischen Möglichkeiten der NPM-Maschinen ergibt sich unter anderem die Fragestellung nach neuen effektiven Messstrategien von Nanostrukturen in extrem großen Messfeldern. Messaufgaben in der Mikro- und Nanotechnologie können sich durch zehntausende Mess- und Prüfmerkmale auszeichnen. Das bedeutet, dass teilweise $10^{6}$ bis $10^{8}$ Messpunkte für die messtechnische Analyse erforderlich sind. Neben neuen Herausforderungen an die Software zur Verarbeitung riesiger Datenmengen wird die Notwendigkeit der Messdaten- und Informationsfusion immer wichtiger.

Es ist sicherlich nicht möglich und auch nicht erforderlich, Nanostrukturen flächendeckend in einer Fläche von $25 \mathrm{~mm} \times 25 \mathrm{~mm}$ mit einer lateralen Auflösung von $10 \mathrm{~nm}$ zu erfassen, und geometrisch zu analysieren. Dabei würden 880 Terabyte Messdaten entstehen. Für die notwendige Scanlänge von ca. 
$62 \mathrm{~km}$ würde ein Rastersondenmikroskop mit einer Scangeschwindigkeit von $10 \mu \mathrm{m} / \mathrm{s}$ ca. $200 \mathrm{Jahre}$ benötigen. Es ist allerdings denkbar, dass eine Vielzahl kleiner Scanflächen (100 $\mu \mathrm{m} \times 100 \mu \mathrm{m})$ in dieser großen Fläche gemessen werden, wobei der örtliche Bezug zwischen den Einzelflächen mit Nanometerpräzision zueinander bekannt sein muss. Geht man einen Schritt weiter, so müssen diese kleinen Scanflächen durch entsprechende Vormessungen entsprechend bestimmter geometrischer Eigenschaften zunächst ermittelt werden. Wie bereits in [12] demonstriert wurde, ist es möglich, dass mit Hilfe des CCD-Kameramikroskops der NPM-Maschine ein Gesamtübersichtsbild der Gesamtmessfläche durch sukzessive Bildaufnahme und nanometergenauem Zusammenfügen der Einzelkamerabilder erstellt werden kann. Eine Gesamtfläche von $25 \mathrm{~mm}$ x $25 \mathrm{~mm}$ kann auf diese Weise in weniger als $30 \mathrm{~min}$ mit ca. 2500 Einzelbildern aufgenommen werden, wobei die laterale Auflösung auf Grund der optischen Messung bei 0,5-1 $\mu \mathrm{m}$ liegt. Das Aneinanderfügen (Stitching) der Einzelbilder wird im Gegensatz zu konventionellen Stitchingverfahren auf der Basis von Bildverarbeitungsalgorithmen mit interferometrischer Genauigkeit erfolgt. Im Anschluss können durch einen Segmentierungsalgorithmus interessierende Flächenbereiche ermittelt und mittels des Rastersondenmikroskops hochauflösend gescannt werden.

Inzwischen konnte auch die großflächige Messung mit dem Weißlicht-Interferenzmikroskop erfolgreich in der NPM-Maschine demonstriert werden (vgl. Abbildung 7). Hier wurden in einem Bereich von $25 \mathrm{~mm}$ x 14,3 mm automatisch etwa 2400 Weißlichtinterferenz-Mikroskopmessungen durchgeführt, die 3D-Daten ermittelt und diese Einzelfelder zu einem Gesamt-3D-Datensatz, bestehend aus 2,6 Milliarden Messpunkten (36 Gigabyte), zusammengesetzt werden.

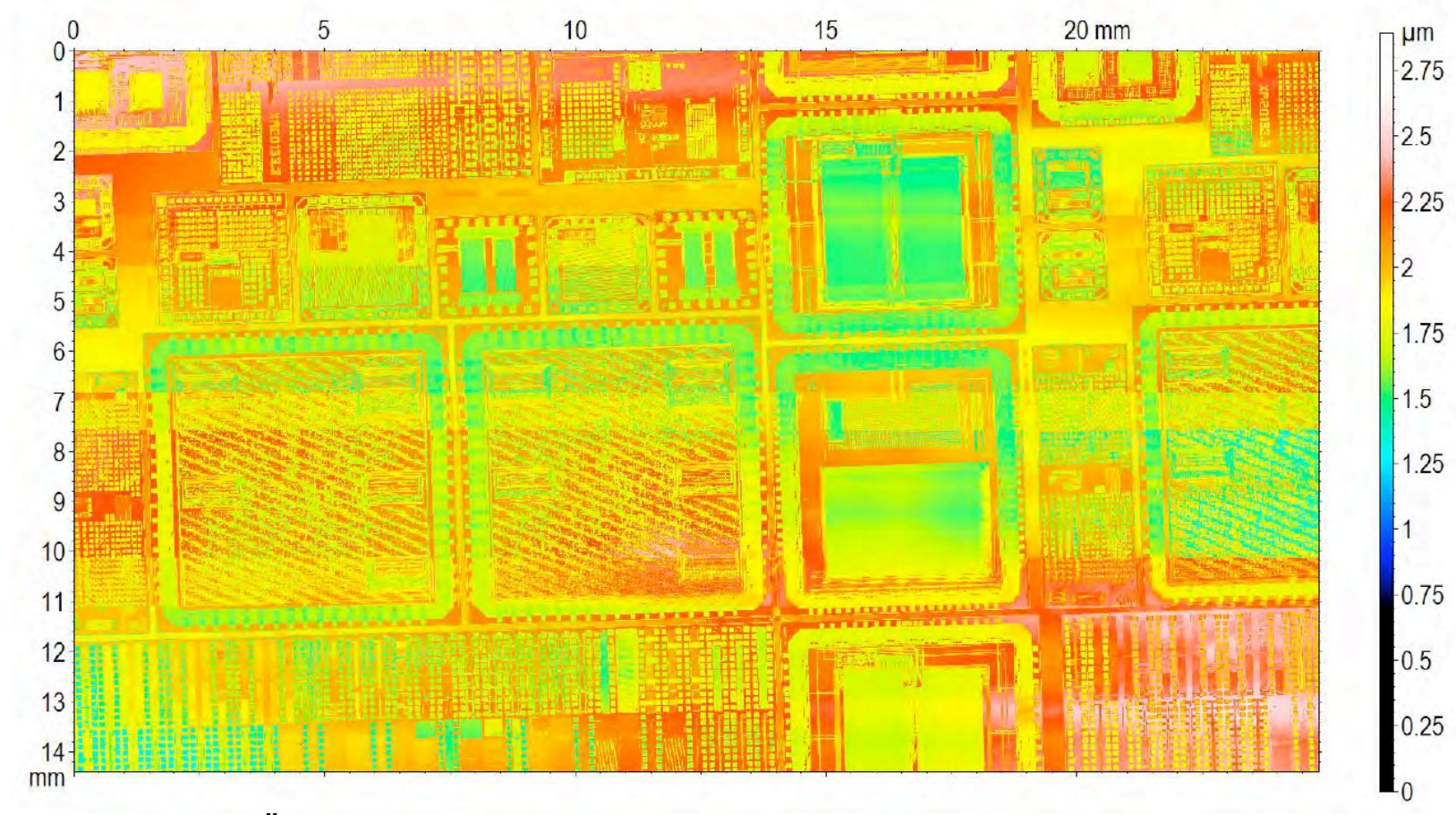

Abbildung 7: 3D-Übersichtsbild mittels Weißlichtinterferenz

Für die Messwertaufnahme wurden etwa 16 Stunden benötigt. Die Messzeit wird hier insbesondere durch die Bildrate der CCD-Kamera von 45 Bildern pro Sekunde begrenzt. Hier sind bereits wesentlich schnellere Kameras mit 200 fps verfügbar (daraus ergibt sich eine Aufnahmezeit von 3,5 h). Die parallele pixelweise Interferogrammauswertung erfolgt mit Hilfe einer Graphic Prozessor Unit und benötigt dadurch lediglich 1 Sekunde Rechenzeit [13]. Als nächster Schritt soll die Umsetzung einer automatischen Bildanalyse mittels Segmentierung demonstriert werden, die beispielsweise die Voraussetzung für eine automatische Prüfplanerstellung bilden kann. Auf Grundlage der Segmentierung können aber wiederum weitere Messungen mit Hilfe der Multisensoranordnung initialisiert werden. 


\section{Bearbeitungstools}

Die Nanopositionier- und Nanomessmaschine erlaubt auch die Integration von Nanotools. Als Alternative zur maskenbasierten Photolithographie werden Nanostrukturierungsverfahren auf der Basis von Rastersondenmikroskopen favorisiert. In [14] wird eine „real-time nanofabrication with high-speed AFM“ in einem Feld von lediglich $2 \mu \mathrm{m} \times 2 \mu \mathrm{m}$ demonstriert. Dagegen ist mit der jetzigen NPM-Maschine prinzipiell eine Nanostrukturierung in einem Feld von $25 \mathrm{~mm}$ x $25 \mathrm{~mm}$ möglich. Dazu wurde ein NT-MDTRastersondenmikroskop „Solver HV-2“ in die NPM-Maschine integriert (vgl. Abbildung 9).

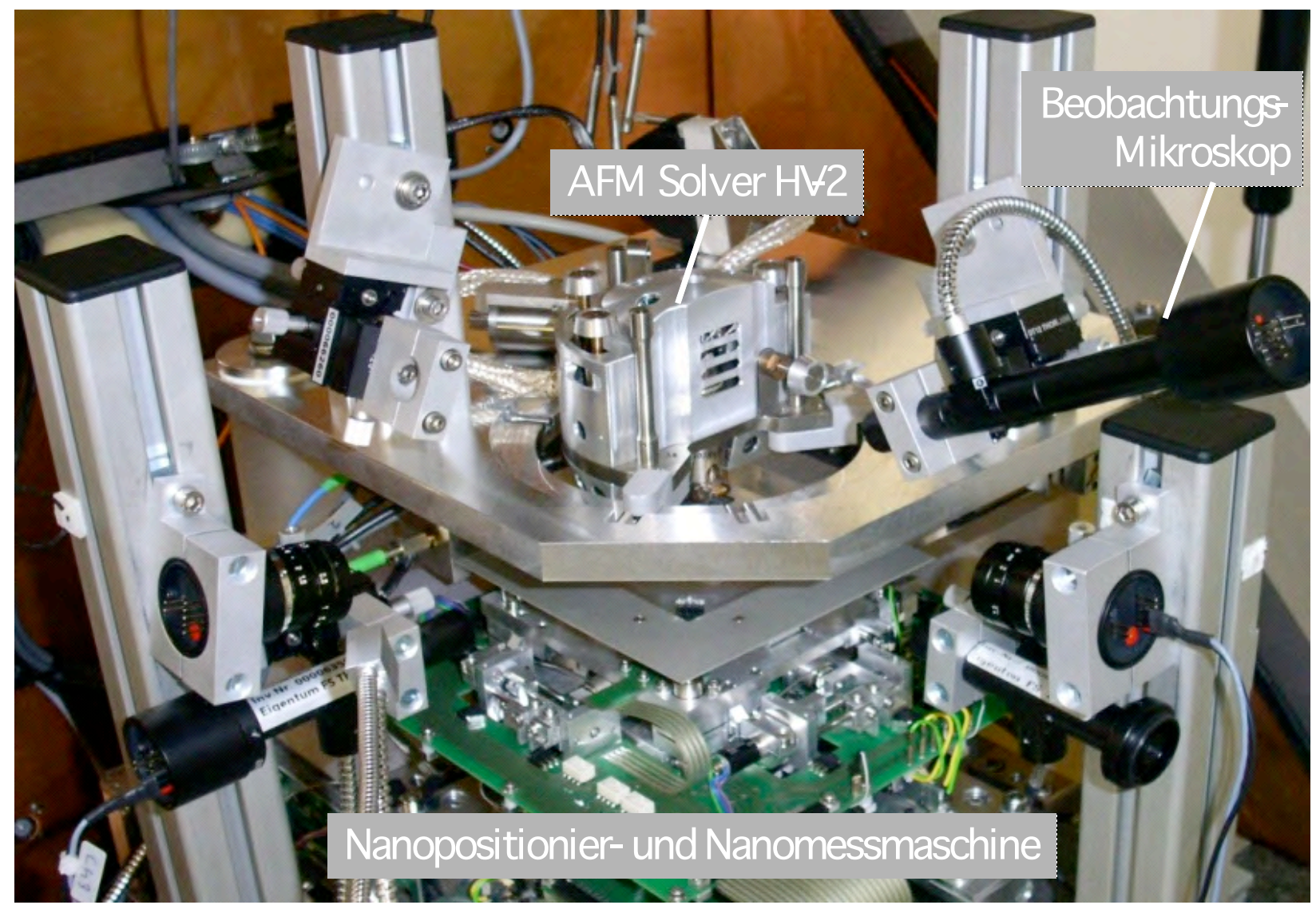

Abbildung 9: AFM Solver HV-2 in NPM-Maschine integriert

Zwei unterschiedliche Verfahren können mit diesem AFM realisiert werden: die Scratching-Lithographie und die Oxydations-Lithographie. Bei ersterem erfolgt die Strukturierung z.B. von Photoresist direkt mit der AFM-Nadel (vgl. Abbildung 7).

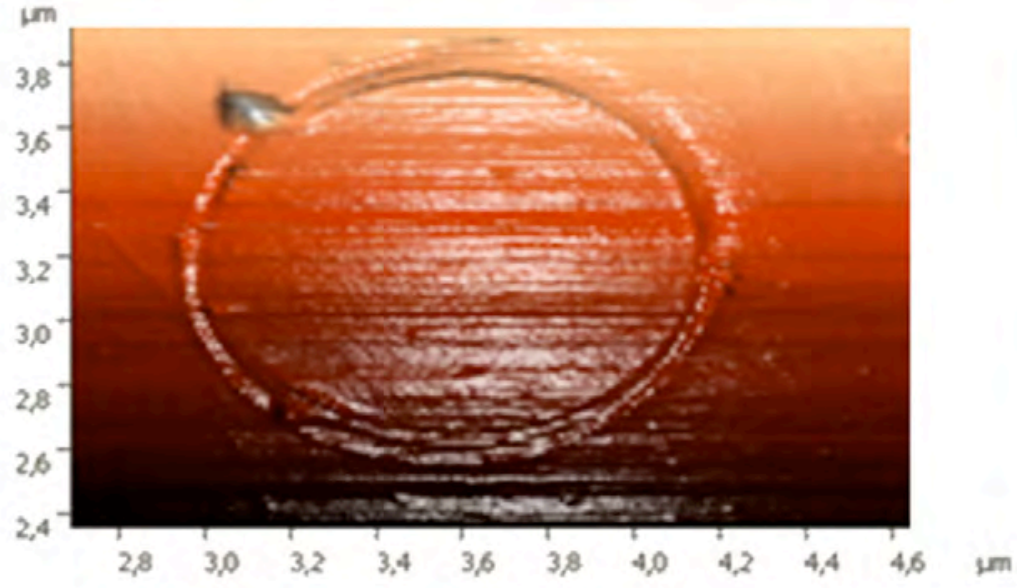

Abbildung 7: Scratching_Lithograpie mittels AFM
Bei dem zweiten Verfahren wird die Siliziumoberfläche durch ein elektrisches Feld zwischen Cantilever-Spitze und SiliziumOberfläche in Anwesenheit von einer dünnen Wasserschicht auf dem Substrat lokal zu Siliziumoxid umgewandelt (vgl. Abbildung 8). Das Ergebnis der Strukturierung kann sich in beiden Fällen direkt nach erfolgter "Bearbeitung" durch einen zweiten AFM-Scan angesehen werden.

Nach der ersten erfolgreichen Demonstration sollen die 


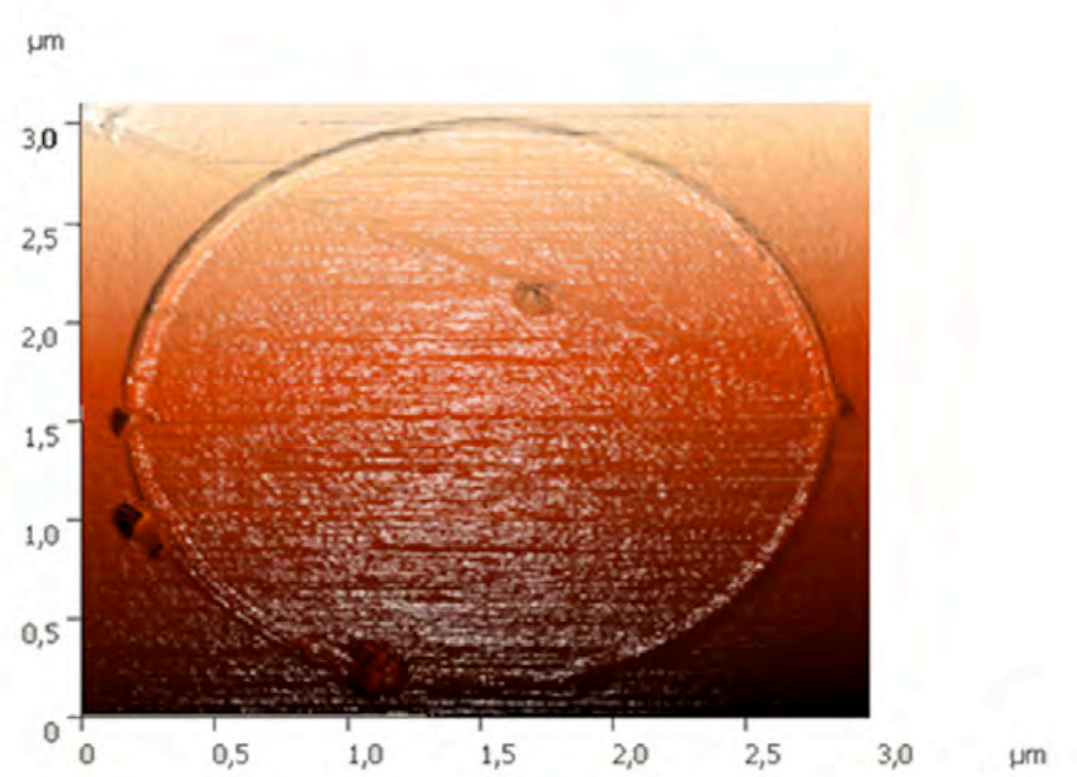

Abbildung 8: Oxydations-Lithographie mittels AFM
Betriebsparameter der beiden Verfahren in der NPMMaschine so optimiert werden, dass eine Strukturierung in großen Arbeitsbereichen mit möglichst kleinen Linienbreiten erfolgen kann. Die Oxydationstechnologie scheint wesentlich besser geeignet zu sein, da der Oxydationsprozess mit geringsten Andruckkräften erfolgen kann. Diese Bearbeitungstechnologie stellt ein großes Potenzial beispielsweise für die Herstellung von NanoimprintStempeln in einem Feld von $25 \mathrm{~mm} \times 25 \mathrm{~mm}$ dar.

\section{Zusammenfassung}

Nanopositionier- und Nanomessmaschinen sind in der Lage, in großen Messbereichen mit Nanometerpräzision zu arbeiten. Durch den Einsatz verschiedener optischer, taktiler und Rastersondensensoren in einem Mikroskoprevolver ist die Möglichkeit für eine Multisensoranwendung gegeben. Damit können multiskalige Messaufgaben in endlicher Zeit durch eine Kombination von verschiedenen Messprinzipien verwirklicht werden. Neben schnellen parallelen optischen Array-Sensoren mit vergleichsweise niedriger lateraler Auflösung kommen lateral hochauflösende Rastersondentechniken in kleinen Messfeldern zum Einsatz.

Durch den Einsatz von Nanotools kann die NPM-Maschine auch als Nanobearbeitungsplattform eingesetzt werden. Erste Ergebnisse konnten mit Hilfe von AFM-Scratching- und AFMOxydationstechniken gezeigt werden.

Damit besitzt die Nanopositionier- und Nanomesstechnik ein großes Potenzial sowohl für die Umsetzung komplexer Messaufgaben als auch für alternative Nanobearbeitungsverfahren in großen Bearbeitungsbereichen, die durch verschiedenste technologische Herausforderungen heute, aber immer mehr auch zukünftig, gestellt werden.

\section{Literatur}

[1] "International Technology Roadmap for Semiconductors, 2009 Edition, Metrology," http://www.itrs.net/links/2009ITRS/2009Chapters 2009Tables/2009 Metrology.pdf (2009)

[2] K. Bobzin, N. Bagcivan, S. Theis, C. Hartmann, J. Holtkamp, A. Gillner, E.H.W. Michaeli, C. Hopmann, S. Eilbracht, M. Schongart, M. Scharf: Manufacturing of micro-structured parts for mass production purposes. In: Proceedings 2011 IEEE International Symposium on Assembly and Manufacturing (ISAM 2011) Pages: 8 pp. (2011)

[3] E. Abbe, „Messapparate für Physiker,“ In: Zeitschrift für Instrumentenkunde, 10(1890)12, pp. 446-448 (1890)

[4] J. Kramar: Nanometre resolution metrology with the Molecular Measuring Machine. In: Meas. Sci. Technol. 16 2121-28 (2005)

[5] R.J. Hocken, D.L. Trumper, C. Wang: Dynamics and control of the UNCC/MIT sub-atomic measuring machine. In: CIRP ANNALS-MANUFACTURING TECHNOLOGY, Volume: 50 Issue: 1 Pages: 373-376 DOI: $10.1016 / S 0007-8506(07) 62143-X(2001)$ 
[6] G. Jäger, E. Manske, T. Hausotte, H.-J. Büchner: The metrological basis and operation of nanopositioning and nanomeasuring machine NMM-1. In: Technisches Messen, 76(2009)5, S. 227 - 234. (2009)

[7] H.A.M. Spaan, R.L. Donker, I. Widdershoven: Isara 400: Development of an ultraprecision CMM for 3D measurement of large parts In: Proc. ASPE spring meeting (2009)

[8] E. Manske, T. Hausotte, R. Mastylo, T. Machleidt, K.-H. Franke, G. Jäger: New applications of the nanopositioning and nanomeasuring machine by using advanced tactile and non-tactile probes. In: Measurement Science and Technology 18, S. 520 - 527 (2007)

[9] N. Vorbringer-Dorozhovets, T. Hausotte, E. Manske, J.C. Shen, G. Jäger: Novel control scheme for a high-speed metrological scanning probe microscope. In: Measurement Science and technology Volume: 22 Issue: 9 Article Number: 094012 DOI: 10.1088/0957-0233/22/9/094012. (2011)

[10] E. Manske, G. Jäger, T. Hausotte, H.-J. Büchner, R. Grünwald, R. FüßI: Konsistenz im Nanometerbereich: Messtechnischer Vergleich von optischen, taktilen und AFM-Sensoren. In: Sensoren und Messsysteme 2008, VDI-Berichte 2011, S. 135-144 (2011)

[11] M. Ritter, M. Hemmleb, O. Sinram, J. Albertz, H. Hohenberg: A versatile 3D calibration object für various micro-range measurement methods. In: Proceedings of ISPRS Congress, $12-23$ July 2004 , Istanbul, Volume XXXV Part 5, pp. 696-701 (2004)

[12] T. Machleidt, E. Sparrer, N. Dorozhovets, E. Manske, et al.: Navigation in a Large Measurement Volume by Using AFM Technology as a Sensor System in the NPMM. In: Technisches Messen, Vol. 76, p. 274-277 (2009)

[13] A. Pacholik, M. Müller, W. Fengler, T. Machleidt, K.-H. Franke: GPU vs FPGA: Example Application on White Light Interferometry. In: International Conference on Reconfigurable Computing and FPGAs, Cancun 30.11-02.12.2011, S. 481-486 (2011)

[14] J.A. Vicary, M.J. Miles, H.H. Wills: Real-time nanofabrication with high-speed atomic force microscopy. In: Nanotechnology 20 (2009) 095302 (5pp) doi:10.1088/0957-4484/20/9/095302 (2009) 